\title{
Influencia de los coadyuvantes tecnológicos utilizados en el proceso de elaboración del aceite de oliva sobre la cinética del proceso de digestión anaerobia del alpechín
}

\author{
Por R. Borja Padilla, J. Alba Mendoza y M. ․ M. Durán Barrantes \\ Instituto de la Grasa y sus Derivados \\ Avda. Padre Garcia Tejero, 4 - 41012 Sevilla
}

\section{RESUMEN}

Influencia de los coadyuvantes tecnológicos utilizados en el proceso de elaboración de aceite de oliva sobre la cinética del proceso de digestión anaerobia del alpechín.

Se ha efectuado un estudio cinético del proceso de digestión anaerobia de un alpechin obtenido con el coadyuvante tecnológico "Olivex" (Carbohidrasa -pectinasas, celulasas y hemicelulasas- procedente de Aspergillus aculeatus) en comparación con un testigo obtenido sin esta formulación enzimática. El proceso se ha realizado en biorreactores con microorganismos inmovilizados en dos soportes micronizados arcillosos, Sepiolita y Bentonita.

A partir de los datos volumen de metano-tiempo, se calculan las constantes especificas de velocidad, Ko, utilizando la ecuación de Roediger. Dentro del rango de concentración de sustrato estudiado se observa una disminución de la constante cinética al aumentar el volumen de residuo añadido a los digestores lo que confirma la existencia de un proceso de inhibición, que es más acusado en el caso del alpechin obtenido con Olivex. Para determinar las constantes de inhibición del proceso se utiliza el modelo propuesto por Levenspiel.

PALABRAS-CLAVE: Alpechin - Cinética - Digestión anaerobia - Olivex.

\section{SUMMARY}

Influence of the technological helpers used in the olive oil elaboration process on the kinetics of the anaerobic digestion of olive mill wastewater.

A kinetic study of the anaerobic digestion of olive mill wastewater, which was obtained with the technological helper "Olivex" (Carbohydrase -pectinases, cellulases and hemicellulases- from the Aspergillus aculea$t u s$ ) was carried out. An identical wastewater, obtained without this enzymatic formulation was also used. The process was carried out in bioreactors with microorganisms immobilized on two micronized clay supports, Sepiolite and Bentonite.

The methane volume-time data pairs obtained were used to calculate the specific rate constant, Ko, by using the Roediger's equation. A decrease of the specific rate constant value was observed over the substrate concentration studied when the volume of wastewater added was increased; this confirmed the occurence of an inhibition process, which was more marked for the olive mill wastewater obtained with Olivex. The Levenspiel's model was used to obtain the inhibition constants of this process.

KEY.WORDS: Anaerobic digestion - Kinetics - Olive mill wastewater-Olivex.

\section{INTRODUCCION}

Los aceites vírgenes de oliva de calidad, se obtienen generalmente a lo largo de las dos primeras épocas de recolección de la campaña olivarera, siempre que el tiem- po transcurrido entre la cogida de los frutos y su elaboración no sobrepase los dos-tres días (Camera, 1978 y Montedoro, 1984). En estas circunstancias las aceitunas suelen presentar ciertas dificultades de comportamiento, durante los diferentes procesos a que son sometidas para la extracción del aceite, debidas básicamente a su constitución bioquímica.

Estos problemas tecnológicos se manifiestan por una característica fluencia de las pastas de aceitunas molidas, que presentan un bajo grado de extractabilidad de aceite, debido generalmente a la formación de emulsiones de las fases líquidas, a la presencia de sólidos finos y a la falta de textura que dificulta su operatividad (Alba, 1982). En los últimos años se han efectuado estudios encaminados a mejorar la tecnología de las aceitunas productoras de "pastas fluentes", con el fin de optimizar los procesos de elaboración y obtener un aumento del rendimiento en aceite y de la calidad del mismo (Leone, 1977).

Otros efectos positivos que se consiguen con la utilización de coadyuvantes tecnológicos en elaiotecnia pueden encontrarse en citas bibliográficas de otros autores (Montedoro, 1987; Alba, 1987a; Alba, 1987b; Alba, 1987c; Giovacchino, 1991).

El objetivo de este trabajo es estudiar la influencia de la utilización del coadyuvante tecnológico constituido por la formulación enzimática "Olivex" sobre la cinética del proceso de digestión anaerobia del alpechín, respecto a uno testigo o de referencia obtenido en ausencia de este producto enzimático. Este proceso de fermentación anaerobia o biometanización se realiza utilizando dos soportes arcillosos de diferente composición química para la inmovilización de los microorganismos responsables del mismo; de esta manera se estudia la influencia del soporte sobre las constantes biocinéticas del proceso.

\section{PARTE EXPERIMENTAL}

\subsection{Productos}

Las aceitunas utilizadas en los ensayos fueron de la variedad "Carrasqueña" procedentes del Valle del Jerte (Cáceres) con índice de maduración de 4,2. 
Como coadyuvante tecnológico se empleó la formulación enzimática Carbohidrasa (pectinasas, celulasas y hemicelulasas) procedente de Aspergillus aculeatus, denominada comercialmente "Olivex", autorizada por el Ministerio de Sanidad y Consumo en el B.O.E. n. ${ }^{\circ} 301$, del 16 de Diciembre de 1989, de la firma Novo Nordisk, S.A. Los ensayos se realizaron con una dosis de $200 \mathrm{~g} / \mathrm{t}$ de "Olivex" diluidos en 8 litros de agua a temperatura ambiente $\left(18-20^{\circ} \mathrm{C}\right)$, agregándose esta solución durante el batido por medio de una bomba dosificadora, en función del caudal de trabajo de la planta. También se obtuvo un alpechín "testigo" ( $\sin$ coadyuvante), con un lote idéntico de aceitunas de la misma variedad.

\subsection{Equipos}

\section{a) Planta Industrial}

Las operaciones de extracción se realizaron en la Almazara Experimental del Instituto de la Grasa, con una planta continua de centrifugación de la marca Pieralisi, modelo SC-45.

\section{b) Equipo de digestión anaerobia}

El equipo usado consiste en dos unidades de digestión anaerobia (UDA) agitadas magnéticamente, de 1 litro de capacidad, sumergidas en un baño de agua a $35^{\circ} \mathrm{C}$. El biogas generado pasa a través de una solución con $\mathrm{NaOH}$ para retener el $\mathrm{CO}_{2}$ producido. El volumen de metano se determinó indirectamente mediante el volumen de agua desplazado por el gas.

Para llevar a cabo este proceso de biometanización, los microorganismos responsables se inmovilizan en el interior del reactor utilizando Sepiolita y Bentonita, silicatos de tamaño micronizado que permiten la fijación de las bacterias anaerobias, con objeto de evitar su pérdida en los efluentes del proceso y con ello una posible disminución de la velocidad del mismo (Borja, 1990).

\subsection{Procedimiento Experimental}

\section{Obtención y toma de muestras de los alpechines}

El plan de trabajo seguido en los ensayos puede resumirse de la siguiente forma:

- El lote de aceitunas seleccionado estuvo constituido por $13.032 \mathrm{~kg}$, que una vez homogeneizado tenía las siguientes características: humedad $48,34 \%$; riqueza grasa 17,24\%; acidez del aceite 0,70.

- Se dividió en dos lotes aproximadamente iguales, para efectuar el ensayo testigo y el tratado, y en ambos se efectuaron las mismas operaciones, consistentes en: efectuar la trituración con una criba de $6 \mathrm{~mm}$ de diámetro, someter la pasta a un termobatido durante un tiempo medio de 90 minutos, alcanzando durante este tiempo una temperatura de $30 \pm 3^{\circ} \mathrm{C}$; bombear a continuación la pasta con un caudal de $900 \mathrm{~kg} / \mathrm{h}$, efectuando en la propia conducción de salida una adición de agua caliente con una temperatura de $42 \pm 3^{\circ} \mathrm{C}$, con objeto de fluidificar la pasta y ponerla en condiciones óptimas de separación. En ambos casos se operó en las condiciones de trabajo normales de este equipo.
- En la salida de alpechines centrifugados se efectuó la toma de muestras de fracciones de $500 \mathrm{ml}$ cada 20 minutos de trabajo, formando un conjunto durante cada ensayo con el que se realizó el estudio de composición y comportamiento, frente a la depuración biológica.

\section{Modo de operar en los reactores anaerobios}

El proceso de digestión anaerobia se inicia utilizando como inóculo una biomasa anaerobia previamente diluida y neutralizada procedente de una balsa de evaporación y lagunaje de alpechín.

A los reactores se añaden inicialmente $750 \mathrm{ml}$ de agua destilada, $250 \mathrm{ml}$ de inóculo y $10 \mathrm{~g}$ del soporte correspondiente. Tras un período de aclimatación de varios meses se realizan una serie de experimentos en régimen discontinuo, añadiendo volúmenes de 20,40 , $60,80,100,120,160$ y $200 \mathrm{ml}$ de alpechín obtenido con Olivex, por un lado. Por otra parte, tras finalizar esta serie de experiencias, se realizan otras similares añadiendo las mismas cargas volumétricas anteriores de alpechín testigo obtenido sin emplear el coadyuvante ya mencionado.

En todos los experimentos, las cargas se añaden después de retirar el mismo volumen de líquido de biorreactor, tras decantar durante 2 horas para evitar pérdidas de biomasa.

En cada experimento se mide el volumen de metano producido diariamente, asi como la DQO inicial y final. La duración de cada experimento se corresponde con el tiempo necesario para la biometanización completa de cada carga. Todos los experimentos se realizan por duplicado.

\section{Características del alpechín utilizado}

Las características del alpechín obtenido con la formulación enzimática Olivex, y del testigo obtenido sin coadyuvante se resumen en la Tabla 1.

Tabla I

Caracteristicas del alpechín obtenido con Olivex y del testigo obtenido sin coadyuvante.

\begin{tabular}{lcc}
\hline & Con “Olivex” & Testigo \\
\hline $\mathrm{pH}$ & 4,7 & 4,8 \\
Demanda Química de Oxígeno $(\mathrm{g} / \mathrm{l})$ & 80 & 70 \\
Acidez Volátil (mg/l) & 550 & 300 \\
Fenoles Totales (ac. cafeico, $\mathrm{mg} / \mathrm{ml})$ & 1,705 & 1,480 \\
Orto-difenoles (ac. cafeico, $\mathrm{mg} / \mathrm{ml})$ & 0,165 & 0,135 \\
Azúcares totales (\%) & 5,1 & 2,4 \\
Nitrógeno total (\%) & 2,3 & 1,6 \\
Sólidos Suspensión Totales (g/l) & 49,1 & 40,9 \\
Sólidos Suspensión Minerales (g/l) & 4,0 & 2,6 \\
Sólidos Suspensión Volátiles (g/l) & 45,1 & 38,3 \\
\hline
\end{tabular}

Los análisis de los parámetros de caracterización de los residuos se realizan de acuerdo con el "Standard Methods for the Examination of Water and Wastewater" (American Public Health Association, 1985). 


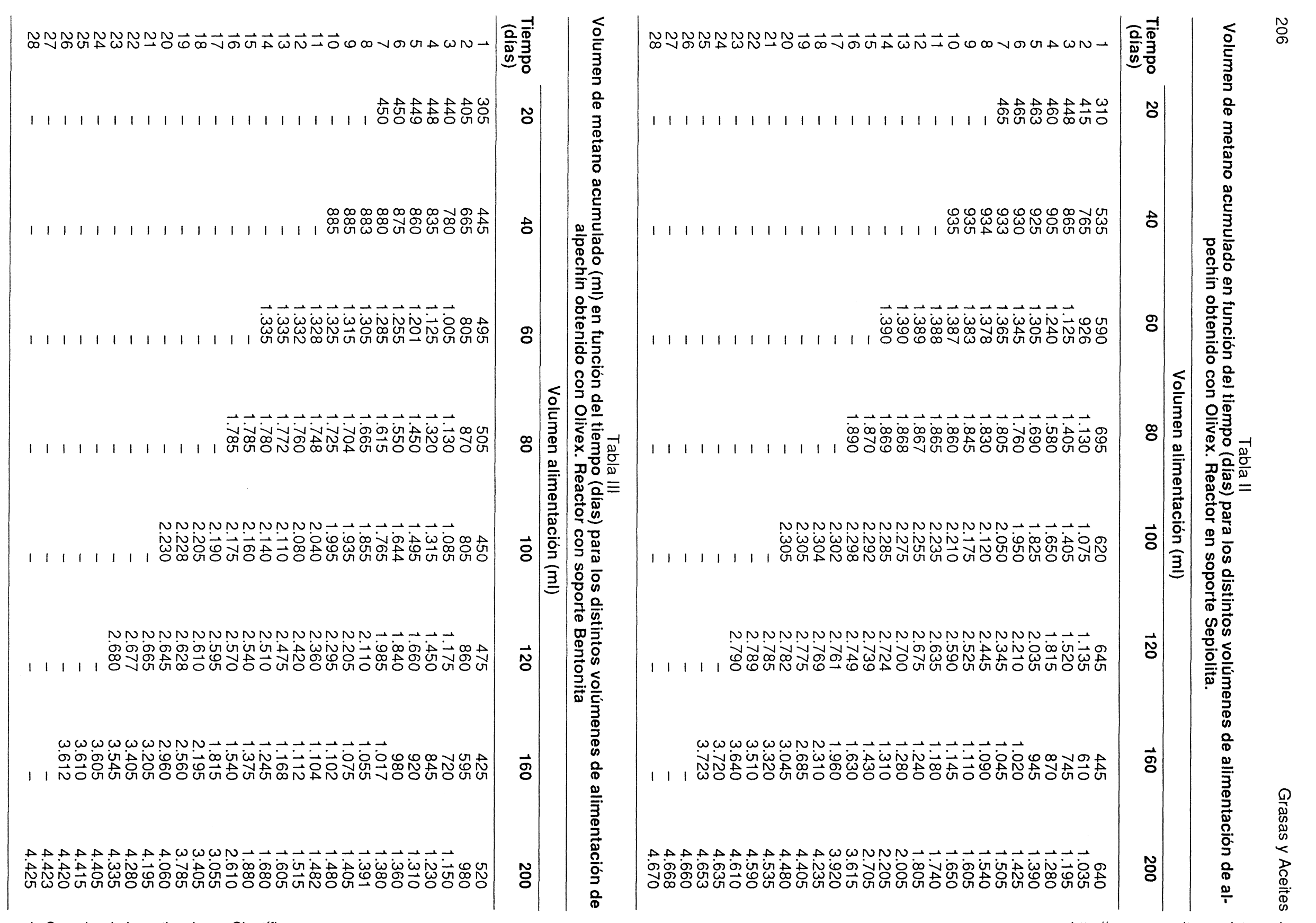




\section{RESULTADOS EXPERIMENTALES}

En las Tablas II, III, IV y V se recogen los valores del volumen de metano acumulado $(\mathrm{ml})$ en función del tiempo (días) para los distintos volúmenes de alimentación utilizados de alpechín obtenido con olivex (II y III) y para el alpechín testigo (IV y V), en los reactores con soporte Sepiolita y Bentonita.

En la Tabla VI aparecen los valores de la DQO al inicio de cada experimento en todos los casos estudiados.

Tabla VI

Valores de la DQO (g/l) al inicio de cada experimento

\begin{tabular}{ccccc}
\hline $\begin{array}{c}\text { Volumen } \\
(\mathrm{ml})\end{array}$ & $\begin{array}{c}\text { Olivex } \\
\text { Sepiolita }\end{array}$ & $\begin{array}{c}\text { Olivex } \\
\text { Bentonita }\end{array}$ & $\begin{array}{c}\text { Testigo } \\
\text { Sepiolita }\end{array}$ & $\begin{array}{r}\text { Testigo } \\
\text { Bentonita }\end{array}$ \\
\hline 20 & 4,55 & 5,05 & 3,85 & 4,35 \\
40 & 6,10 & 6,55 & 5,25 & 5,70 \\
60 & 7,65 & 8,10 & 6,55 & 7,05 \\
80 & 9,15 & 9,60 & 7,90 & 8,35 \\
100 & 10,70 & 11,15 & 9,25 & 9,70 \\
120 & 12,25 & 12,70 & 10,60 & 11,05 \\
160 & 15,35 & 15,75 & 13,30 & 13,70 \\
200 & 18,40 & 18,80 & 16,00 & 16,40 \\
\hline
\end{tabular}

\section{DISCUSION DE LOS RESULTADOS}

A partir de los valores puntuales del volumen de metano acumulado en función del tiempo y admitiendo que globalmente el proceso de fermentación anaerobia sigue una cinética de primer orden (Grujer, 1983; Winkler, 1983), se obtienen las constantes específicas de velocidad para cada experimento realizado, mediante la ecuación de Roediger (Edeline, 1980):

$$
G=G_{m}\left[1-\exp \left(-K_{o} t\right)\right]
$$

Siendo:

$G=$ volumen de metano acumulado en función del tiempo $(\mathrm{ml})$.

$\mathrm{G}_{\mathrm{m}}=$ volumen máximo de metano acumulado (ml) considerando un tiempo de digestión infinito.

$\mathrm{K}_{\mathrm{o}}=$ constante específica de velocidad (días ${ }^{-1}$ ).

$\mathrm{t}=$ tiempo de digestión (días).

Para poder aplicar a los resultados experimentales el modelo formulado se debe comprobar, en primer lugar, que los datos $(G, t)$ se ajustan a la ecuación propuesta. A título de ejemplo en las figuras 1 y 2 se representan los valores de $\mathrm{G}(\mathrm{ml})$ en función del tiempo (días) para algunos experimentos realizados con alpechín obtenido con olivex y con el testigo respectivamente en el reactor con sepiolita.

\section{Olivex}

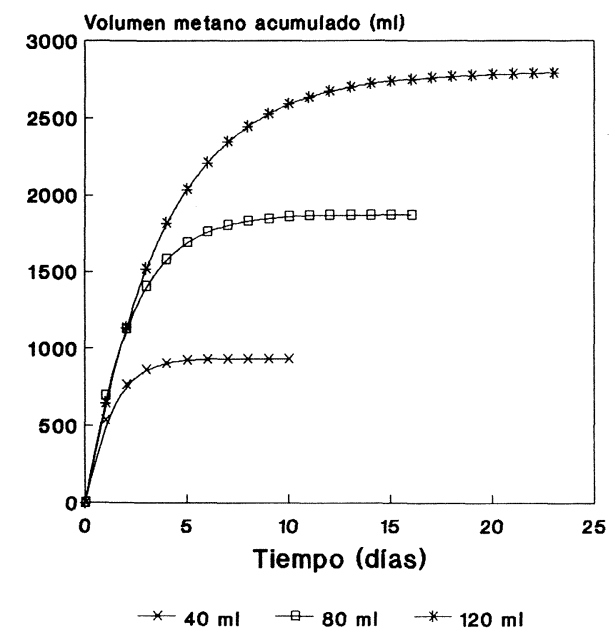

Figura 1

Volumen de metano acumulado ( $\mathrm{ml}$ ) en función del tiempo (dias), para algunas cargas volúmetricas de alpechin obtenido con Olivex (reactor Sepiolita).

Se comprueba en todos los casos, que para volúmenes de alimentación comprendidos entre 20 y $120 \mathrm{ml}$ de alpechín, las curvas cinéticas cumplen las características exigibles a la ecuación propuesta. Así, para $t=0, G=0$ y cuando $t$ tiende a infinito, $G=G_{m}$-se alcanza una asíntota- lo que se observa en las gráficas; finalmente, la pendiente de las curvas, en cada caso, decrece al aumentar el tiempo y no se observan puntos singulares. Estas razones, justifican el ajuste de los datos experimentales al modelo propuesto, en estos casos.

\section{Testigo}

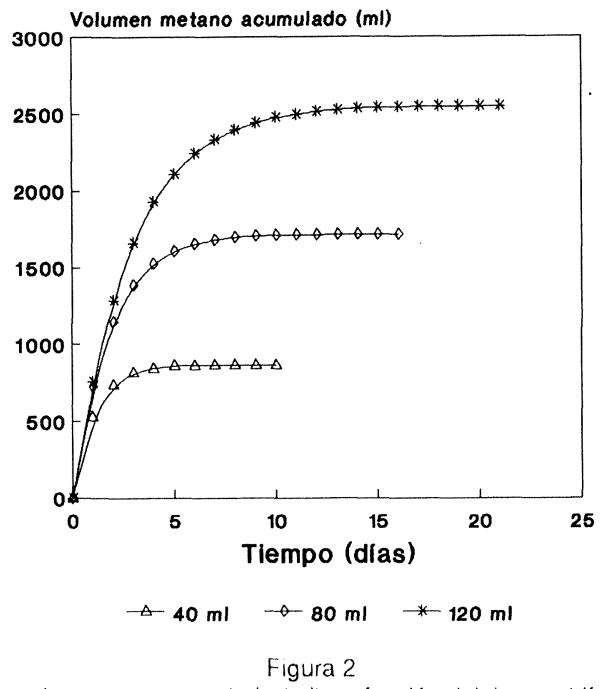

Volumen de metano acumulado (ml) en función del tiempo (dias), para algunas cargas volumétricas de alpechin testigo (reactor Sepiolita). 
Sin embargo, para cargas volumétricas superiores (160 y $200 \mathrm{ml}$ ), las curvas $\mathrm{G}=\mathrm{f}(\mathrm{t})$ ya no se ajustan a un modelo de tipo exponencial, tal como predice la ecuación de Roediger, sino que presentan tramos de escasa o nula producción de metano, lo que determina la existencia de algunos puntos de inflexión en las curvas correspondientes. En las figuras 3 y 4 aparecen a título de ejemplo estas curvas cinéticas correspondientes al reactor con Sepiolita para los casos del alpechín obtenido con olivex y para el de referencia respectivamente. Este mismo comportamiento se observa en el reactor con soporte Bentonita.

\section{Olivex}

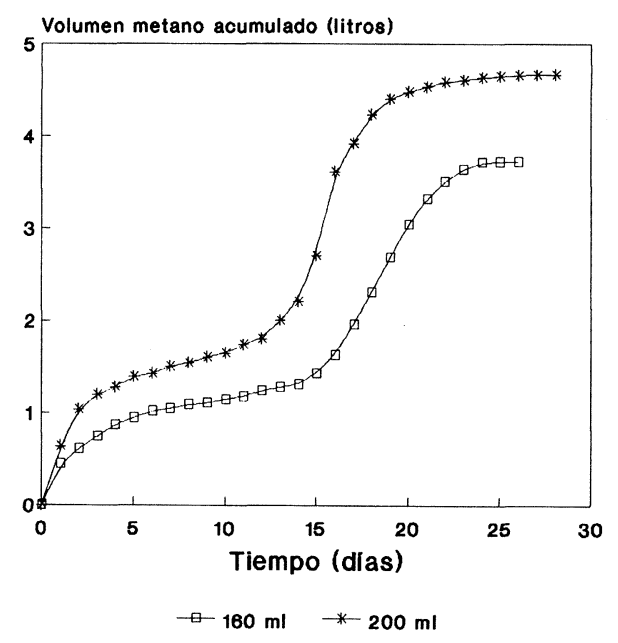

Figura 3

Volumen de metano acumulado ( $\mathrm{ml}$ ) en función del tiempo (dias), para las cargas volumétricas de 160 y $200 \mathrm{ml}$ de alpechín obtenido con Olivex (reactor Sepiolita).

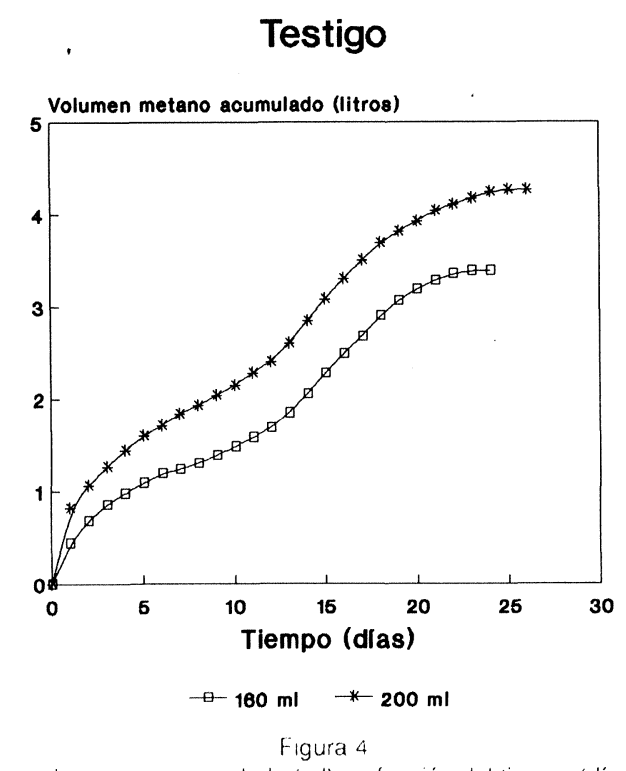

Volumen de metano acumulado ( $\mathrm{ml}$ ) en función del tiempo (dias), para las cargas volumétricas de 160 y $200 \mathrm{ml}$ de alpechin testigo (reactor Sepiolita)
La determinación de la constante específica de velocidad, $\mathrm{K}_{0}$, a partir de los datos experimentales, en el rango de concentración de sustrato donde el modelo es aplicable (cargas 20 a $120 \mathrm{ml}$ de ambos alpechines), se realiza numéricamente mediante ordenador utilizando el Software TSP International versión 4.OD (Stanford CA, 94306 USA). En la Tabla VII se resumen los valores de esta constante cinética junto con sus límites de confianza (considerando un intervalo de probabilidad del 95\%).

Tabla VII

Valores de la constante específica de velocidad, Ko (días ${ }^{-1}$ ), con sus límites de confianza para cada reactor y experimento realizado.

\begin{tabular}{ccccc}
\hline $\begin{array}{c}\text { Volumen } \\
(\mathrm{ml})\end{array}$ & $\begin{array}{c}\text { Olivex } \\
\text { Sepiolita }\end{array}$ & $\begin{array}{c}\text { Olivex } \\
\text { Bentonita }\end{array}$ & $\begin{array}{c}\text { Testigo } \\
\text { Sepiolita }\end{array}$ & $\begin{array}{c}\text { Testigo } \\
\text { Bentonita }\end{array}$ \\
\hline 20 & $1,10 \pm 0,02$ & $1,13 \pm 0,01$ & $1,19 \pm 0,02$ & $1,26 \pm 0,01$ \\
40 & $0,85 \pm 0,01$ & $0,70 \pm 0,02$ & $0,95 \pm 0,02$ & $0,81 \pm 0,02$ \\
60 & $0,55 \pm 0,01$ & $0,46 \pm 0,01$ & $0,64 \pm 0,01$ & $0,55 \pm 0,01$ \\
80 & $0,46 \pm 0,01$ & $0,33 \pm 0,01$ & $0,55 \pm 0,01$ & $0,44 \pm 0,01$ \\
100 & $0,31 \pm 0,01$ & $0,22 \pm 0,01$ & $0,40 \pm 0,01$ & $0,30 \pm 0,01$ \\
120 & $0,26 \pm 0,01$ & $0,19 \pm 0,01$ & $0,35 \pm 0,01$ & $0,30 \pm 0,01$ \\
\hline
\end{tabular}

Como se observa en la Tabla VII, en los dos alpechines utilizados y para los dos soportes empleados existe una drástica disminución de la constante específica de velocidad, $\mathrm{K}_{\mathrm{o}}$, con la concentración de sustrato, lo que da idea de la existencia de un proceso de inhibición. Este retardo en la cinética del proceso es ligeramente más acusado en el reactor con soporte Bentonita en los dos residuos estudiados.

\subsection{Modelo de Levenspiel para la cuantificación} de la inhibición

Para la modelización del proceso de inhibición se utiliza la ecuación propuesta por Levenspiel (1986), que relaciona la constante específica de velocidad, $\mathrm{K}_{\mathrm{o}}$, con la concentración de sustrato, para el caso de existir un proceso de inhibición debido a la presencia de una sustancia tóxica.

$$
K_{0}=K\left[1-\left(S / S^{*}\right)\right]^{n}(2)
$$

Donde:

$\mathrm{K}_{\mathrm{o}}=$ constante especifica de velocidad, obtenida al aplicar la ecuación de Roediger (días ${ }^{-1}$ ).

$\mathrm{S}=$ concentración de sustrato media del experimento (g DQO/I).

$\mathrm{S}^{\star}=$ concentración de inhibidor crítica (g DQO/l) a partir de la cual la reacción o actividad de las células se detiene.

$K=$ constante de velocidad en ausencia de inhibición (dias ${ }^{-1}$ ).

$n=$ parámetro de inhibición (adimensional). 
Esta ecuación se puede linealizar tomando la forma:

$$
\operatorname{Ln} K_{0}=\operatorname{Ln} K+n \operatorname{Ln}\left[1-\left(S / S^{*}\right)\right](3)
$$

De tal manera que de las representaciones de los valores del logaritmo neperiano de $\mathrm{K}_{\mathrm{o}}$ frente al logaritmo neperiano de [1-(S/S*)] podemos obtener el valor de la constante $\mathrm{K}$ a través de la ordenada en el origen y $n$ directamente por medio de la pendiente.

La concentración de inhibición crítica $S^{*}$ se obtiene por tanteo, tomándose el valor, con el que el coeficiente de correlación de las representaciones lineales anteriores sea más elevado, y por tanto el ajuste sea más adecuado.

Por otra parte, para poder encontrar la relación enunciada entre $K_{0}$ y $S$ debe tenerse en cuenta que $K_{0}$ es un valor medio en un rango de concentraciones de sustrato; por tanto a $\mathrm{K}_{\mathrm{o}}$ se asignará el valor medio de $\mathrm{S}$-media aritmética entre la concentración inicial y final, calculado a partir de los datos de la Tabla VI, teniendo en cuenta que la DQO final de los efluentes correspondientes al alpechín con Olivex es 3,0 g/l (Sepiolita) y 3,5 g/l (Bentonita) y la correspondiente a los efluentes del alpechín testigo $2,5 \mathrm{~g} / \mathrm{l}$ (Sepiolita) y $3,0 \mathrm{~g} / \mathrm{l}$ (Bentonita) respectivamente.

La Tabla VIII recoge los valores de $\mathrm{K}$ (días ${ }^{-1}$ ), $\mathrm{n}$ y $\mathrm{S}^{*}$ obtenidos por el procedimiento mencionado anteriormente.

Tabla VIII

Valores de K (días ${ }^{-1}$ ), $n$ y $S^{*}$ (g DQO/I) por cada alpechín utilizado en ambos reactores.

\begin{tabular}{lcrrr}
\hline & $\begin{array}{c}\text { Olivex } \\
\text { Sepiolita }\end{array}$ & $\begin{array}{c}\text { Olivex } \\
\text { Bentonita }\end{array}$ & $\begin{array}{c}\text { Testigo } \\
\text { Sepiolita }\end{array}$ & $\begin{array}{c}\text { Testigo } \\
\text { Bentonita }\end{array}$ \\
\hline $\mathrm{K}$ & 4,10 & 6,21 & 3,51 & 4,77 \\
$\mathrm{n}$ & 15,21 & 16,09 & 15,11 & 15,23 \\
$\mathrm{~S}^{*}$ & 45 & 40 & 45 & 40 \\
\hline
\end{tabular}

Como se ha expuesto, la constante específica de velocidad, $\mathrm{K}_{0}$, disminuye fuertemente al aumentar la concentración de sustrato en todos los casos, lo que prueba la existencia de un fenómeno de inhibición. La mayor variación se observa para el alpechín obtenido con Olivex, comprobándose que la relación $\mathrm{K}_{\mathrm{o}}(20 \mathrm{ml}) / \mathrm{K}_{\mathrm{o}}(120$ $\mathrm{ml}$ ) (Tabla VII) es mayor en este residuo que en el de referencia, hecho que ocurre en los dos reactores utilizados. Esto se encuentra en consonancia con los resultados obtenidos al aplicar el modelo de Levenspiel en el que se obtiene para este alpechín obtenido con enzimas valores más elevados de la constante de velocidad en ausencia de inhibición, $\mathrm{K}$ (Tabla VIII) en los dos digestores empleados.

Por otra parte, el parámetro de inhibición n presenta valores ligeramente más elevados para el alpechín obtenido con Olivex que para el testigo, siendo esta diferencia más acusada en el caso del reactor con soporte Bentonita donde se produce un aumento del orden del $5 \%$.

En lo que se refiere a los valores de $S^{*}$ o concentración de inhibición crítica, es interesante resaltar que estos se encuentran afectados por el soporte utilizado; así en ambos alpechines, el valor de $S^{\star}$ es 45 y $40 \mathrm{~g} \mathrm{DQO/l,}$ para la Sepiolita y Bentonita respectivamente. Esto indica que el digestor con Sepiolita permite trabajar en un rango más elevado de concentraciones de sustrato, sin llegar a alcanzar una total inhibición.

Por último, a partir de los valores obtenidos de $\mathrm{K}, \mathrm{n}$ y $S^{*}$ para cada alpechín y reactor empleado y utilizando la ecuación de Levenspiel (2) se calculan los valores teóricos de la constante especifica de velocidad $\mathrm{K}_{\mathrm{o}}$. En la Tabla IX se recogen los valores teóricos de $\mathrm{K}_{\mathrm{o}}$, junto con el porcentaje de desviación respecto de los valores experimentales (Tabla VII) en todos los casos estudiados.

Como se aprecia, los parámetros biocinéticos obtenidos mediante este modelo, reproducen los valores de la constante especifica de velocidad de este proceso con una desviación inferior al $10 \%$ en más del $75 \%$ de los casos.

Tabla IX

Valores teóricos de Ko (dias-1) junto con el porcentaje de desviación respecto a los valores experimentales para cada caso estudiado.

\begin{tabular}{|c|c|c|c|c|c|c|c|c|}
\hline \multirow{2}{*}{$\begin{array}{l}\text { Experimento } \\
\qquad(\mathrm{ml})\end{array}$} & \multicolumn{2}{|c|}{ Olivex-Sepiol. } & \multicolumn{2}{|c|}{ Olivex-Benton. } & \multicolumn{2}{|c|}{ Test.-Sepiol. } & \multicolumn{2}{|c|}{ Test.-Benton. } \\
\hline & Ko & \%Desv. & & $\%$ Desv. & Ko & \%Desv. & Ko & Desv. \\
\hline 20 & 1,08 & 1,7 & 1,01 & 11,0 & 1,16 & 2,8 & 1,10 & 13,0 \\
\hline 40 & 0,81 & 4,7 & 0,72 & 2,7 & 0,90 & 5,3 & 0,83 & 1,5 \\
\hline 60 & 0,60 & 10,0 & 0,50 & 8,2 & 0,71 & 10,0 & 0,62 & 12,6 \\
\hline 80 & 0,45 & 2,2 & 0,35 & 5,7 & 0,55 & 0,0 & 0,46 & 5,5 \\
\hline 100 & 0,33 & 7,1 & 0,24 & 9,6 & 0,42 & 5,7 & 0,34 & 11,7 \\
\hline 120 & 0,24 & 6,5 & 0,16 & 13,8 & 0,33 & 6,9 & 0,25 & 15,7 \\
\hline
\end{tabular}




\subsection{Coeficientes de rendimiento}

Dado que para cada experimento realizado se conoce el volumen de metano producido, $G$, así como la DQO inicial y final, es posible calcular el coeficiente de rendimiento $Y_{p / s}$ para cada uno de los casos estudiados. A partir de un ajuste lineal de las parejas de valores (G. DQO consumida) mediante el método de mínimos cuadrados, se obtienen a través de la pendiente los siguientes valores de este coeficiente de rendimiento con sus límites de confianza, considerando un intervalo de probabilidad del $95 \%$ :

Alpechín obtenido con Olivex:

- Reactor con Sepiolita: $303 \pm 2 \mathrm{ml} \mathrm{CH}_{4} / \mathrm{g}$ DQO.

- Reactor con Bentonita: $290 \pm 1 \mathrm{ml} \mathrm{CH}_{4} / \mathrm{g}$ DQO.

Alpechín testigo:

- Reactor con Sepiolita: $315 \pm 2 \mathrm{ml} \mathrm{CH}_{4} / \mathrm{g} \mathrm{DQO}$.

- Reactor con Bentonita: $306 \pm 2 \mathrm{ml} \mathrm{CH}_{4} / \mathrm{g} \mathrm{DQO}$.

Como se observa, el valor de este coeficiente es algo mayor para el alpechín testigo en relación con el obtenido con coadyuvante. Este resultado está en consonancia con la secuencia obtenida para las constantes cinéticas del proceso, lo que demuestra que el residuo obtenido con Olivex, con un mayor contenido en compuestos fenólicos (de marcado efecto inhibidor) y en materia orgánica en general presenta mayores dificultades para la depuración vía anaerobia, dando lugar a un menor rendimiento.

En cuanto al comportamiento de los soportes ensayados, se aprecia un mayor coeficiente de rendimiento en el reactor con Sepiolita en relación a la Bentonita. Este hecho está de acuerdo con resultados precedentes (Borja, 1990) que revelan el comportamiento favorable de silicatos magnésicos de tipo sepiolítico como soporte de inmovilización, dadas sus características peculiares para favorecer el crecimiento y desarrollo de las bacterias metanogénicas, responsables directas del proceso anaerobio.

\section{CONCLUSIONES}

1. A partir de los datos experimentales volumen de metano acumulado $(G)$, tiempo $(t)$, mediante la ecuación de Roediger se obtienen las constantes específicas de velocidad del proceso de digestión anaerobia de un alpechín obtenido con Olivex y de otro testigo sin este coadyuvante.

El modelo solo es aplicable para bajas concentraciones de sustrato (cargas volumétricas de 20 a $120 \mathrm{ml}$ de alpechín).

2. Existe una acusada disminución de las constantes de velocidad con la concentración de sustrato para ambos alpechines, lo que revela la existencia de un proceso de inhibición, que es ligeramente más intenso en el caso del alpechín obtenido con Olivex.

3. Se ha utilizado el modelo de Levenspiel para obtener las constantes cinéticas de inhibición que rigen este proceso, confirmándose el resultado anterior. A partir de ellas se pueden reproducir los valores de las constantes específicas de velocidad, $\mathrm{K}_{\mathrm{o}}$, con un grado de desviación inferior al $10 \%$, en más del $75 \%$ de los casos.

4. El soporte Sepiolita favorece la cinética y aumenta el coeficiente de rendimiento respecto a la Bentonita, observándose este fenómeno en los dos alpechines estudiados.

\section{AGRADECIMIENTOS}

Queremos hacer constar nuestro agradecimiento a D. a Carmen Sánchez González y a D. Francisco Cano Martín por su eficaz ayuda y colaboración.

\section{BIBLIOGRAFIA}

Alba, J.; Muñoz, E.y Martínez, J.M. (1982).- "Obtención de aceite de oliva. Empleo de productos que facilitan su extracción".- Alimentaria 25-55.

Alba Mendoza, J. y Ruiz Gómez, A. (1987a).- "Estudios a nivel de laboratorio e industrial sobre la utilización enzimática en la obtención del aceite de oliva".- Memorias del II World Congress of Food Tecnology IV, 2.885-2.897

Alba Mendoza, J.; Ruiz Gómez, A. y Gutiérrez Rosales, F. (1987 b)."Eficacia de la formulación enzimática Röhament $O$ en la tecnología del aceite de oliva. Composición y valoración organoléptica de los aceites obtenidos".- Grasas y Aceites 38, 271-277.

Alba Mendoza, J. y Ruiz Gómez, A. (1987c).- "Efecto de la formulación enzimática Olivex en el proceso de obtención de aceite de oliva". Actas del VII Congreso Nacional de Química. Química Agricola y Alimentaria-2, III, 297-304.

American Public Health Association (1985)-- Standard Methods for the Examination of Water and Wastewater (16 th edn.).- APHA, Washington, D.C.

Borja, R.; Martin A. y Fiestas, J.A. (1990).- "Estudio cinético de la depuración anaerobia del alpechin en presencia de diversos soportes para la inmovilización de los microorganismos responsables del proceso".Grasas y Aceites 41, 347-356.

Camera, L.; Angerosa, F. y Cucarachi, A. (1978).- "Influenza dello stoccaggio delle olive sui costituenti della frazione sterolica dell'olio".- Riv. Ital. Sost. Grasse, 107-112.

Edeline, F. (1980).- "L'épuration biologique des eaux residuaires. Theórie et Technologie".- Ed. Cebecoc Liege, Belgium.

Giovacchino, L. (1991). "Situation en Italie de la voie enzymatique pour la production d'huile d'olive: résultats des essais et perspectives". Rev. Française des Corps Gras, 85-94.

Grujer, W. and Zehnder, J.B. (1983).- "Conversion process in anaerobic digestion".- Wat. Sci. Tech. 15, 127-167.

Leone, A.M. y Colaboradores (1977).- "L'impiego elaiotecnico di un sistema enzimático pectocellulolitico. Rendimiento in olio e qualita de prodotto".- La Riv. Ital. Sost. Grasse LIV, 514-530.

Levenspiel, O. (1986).- "El omnilibro de los reactores químicos". Ed. Reverté, Barcelona.

Montedoro, G.; Garfolo, L. (1984).- "Caracteristiche qualitative degli oli virgini di oliva. Influenza di alcune variabili: Varietá, ambiente, conservazone, estrazione, condizionamento de prodotto finito".- Riv. Ital Sost. Grasse, 157-168.

Montedoro, G.F. (1987).- "Impiego di preparati enzimatici e drenanti nell'estrazione meccanica di oli di oliva".- La Riv. Ital. Sost. Grasse LXIV, 415-421.

Winkler, M. (1983).- "Biological treatment of Waste-Water".- Elli Horwood Ltd, Publishers. Chapter 4.

(Recibido: Diciembre 1991) 